\title{
Chiral phase transition with $2+1$ quark flavors in an improved soft-wall AdS/QCD model
}

\author{
Zhen Fang, ${ }^{1,2,3, *}$ Yue-Liang $\mathrm{Wu},{ }^{3,4, \dagger}$ and Lin Zhang ${ }^{3,2, \$}$ \\ ${ }^{1}$ Department of Applied Physics, School of Physics and Electronics, Hunan University, \\ Changsha 410082, China \\ ${ }^{2}$ School of Physical Sciences, University of Chinese Academy of Sciences, Beijing 100049, China \\ ${ }^{3}$ CAS Key Laboratory of Theoretical Physics, Institute of Theoretical Physics, \\ Chinese Academy of Sciences, Beijing 100190, China \\ ${ }^{4}$ International Centre for Theoretical Physics Asia-Pacific (ICTP-AP), \\ University of Chinese Academy of Sciences, Beijing 100049, China
}

(Received 3 October 2018; published 7 December 2018)

\begin{abstract}
We study the chiral phase transition with $2+1$ quark flavors in an improved soft-wall AdS/QCD model, which can produce the light meson spectrum and many other low-energy quantities consistent with experiments in the two-flavor case. The chiral transition behaviors of the quark condensates at different quark masses are analyzed in detail, and the $\left(m_{u, d}, m_{s}\right)$ phase diagram for the quark sector has been obtained from the improved soft-wall model. We find that the features of the calculated phase diagram are completely consistent with the standard scenario, which is supported by lattice simulations and theoretical arguments. The evidence of a tricritical point on the $m_{u, d}=0$ boundary of the $\left(m_{u, d}, m_{s}\right)$ phase diagram is first clearly presented in the bottom-up AdS/QCD.
\end{abstract}

DOI: 10.1103/PhysRevD.98.114003

\section{INTRODUCTION}

Study on the phase transition and vacuum structure of quantum chromodynamics (QCD) is very important for our understanding of the low-energy physics of strong interaction and even the evolution of our Universe [1]. It is known that chiral symmetry breaking and quark confinement are two essential features of low-energy QCD. It is widely believed that at high temperature or baryon density QCD will undergo a transition from the hadron matter to the quark-gluon plasma accompanied by the chiral symmetry restoration and color deconfining process. The relation between the chiral and deconfining phase transitions is a hot topic but without a final conclusion up to now.

To characterize QCD phase transitions, we need to choose proper order parameters [2-5]. In this work, we only consider the chiral phase transition with the chiral condensate as an order parameter. The sketched plot in

\footnotetext{
*Corresponding author. fangzhen@itp.ac.cn

ylwu@itp.ac.cn

${ }^{\sharp}$ Corresponding author. zhanglin@itp.ac.cn

Published by the American Physical Society under the terms of the Creative Commons Attribution 4.0 International license. Further distribution of this work must maintain attribution to the author(s) and the published article's title, journal citation, and DOI. Funded by SCOAP.
}

Fig. 1 shows the standard scenario of a QCD phase diagram in the $\left(m_{u, d}, m_{s}\right)$ quark mass plane [6], which gives the quark mass dependence of the order of the phase transition in the $2+1$-flavor case.

In recent decades, the holographic approach, viz., the anti-de Sitter/conformal field theory (AdS/CFT) correspondence [7-9], provides a powerful tool to tackle the lowenergy nonperturbative problems of QCD, which is usually called AdS/QCD or holographic QCD. Extensive research has been born out of this field with the aim of giving good descriptions of nonperturbative phenomena with the strong interaction [10-71]. In this work, we consider the $2+1$ flavor chiral phase transition in an improved soft-wall AdS/ QCD model [26], which has been shown to reproduce the light meson spectrum and many other low-energy quantities consistent with experiments, and also the right chiral transition behavior in the two-flavor case. The magnetic effects have also been studied in this model [41], which displays inverse magnetic catalysis in the chiral transition indicated by lattice simulations [72,73].

The paper will be organized as follows. In Sec. II, we give a brief introduction of the improved soft-wall AdS/ QCD model with two flavors [26]. Then we generalize this model to the case of $2+1$ flavors. In Sec. III, we study the chiral phase transition for $2+1$ quark flavors in the improved soft-wall model and the $\left(m_{u, d}, m_{s}\right)$ phase diagram will be obtained and compared with that shown in Fig. 1. In Sec. IV, we study the parameter dependence of the 


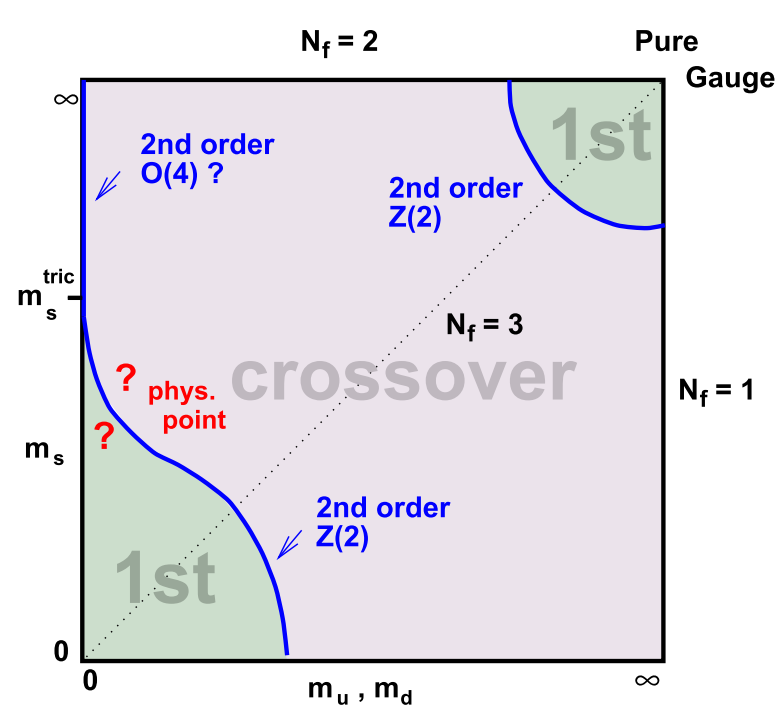

FIG. 1. The expected phase diagram in the quark mass plane $\left(m_{u, d}, m_{s}\right)$ [6]. The lateral axis denotes the up/down quark mass $m_{u, d}$, and the vertical axis denotes the strange quark mass $m_{s}$. The blue curves represent the second-order lines of the phase transition, which divide the phase diagram into three parts. The light green regions denote the first-order phase transition, while the grey part denotes the crossover transition. The diagonal line corresponds to the flavor-symmetric case with $m_{u}=m_{d}=m_{s}$.

$2+1$-flavor chiral transition and the condition in which the standard scenario of the phase diagram exists. In Sec. V, we conclude our work and give a brief discussion of the chiral phase transition in the improved soft-wall model.

\section{THE IMPROVED SOFT-WALL ADS/QCD MODEL}

\section{A. Two-flavor case}

We use the pure $\mathrm{AdS}_{5}$ spacetime as the bulk background with the metric ansatz:

$$
d s^{2}=e^{2 A(z)}\left(\eta_{\mu \nu} d x^{\mu} d x^{\nu}-d z^{2}\right),
$$

where $A(z)=-\ln \frac{z}{L}$ is scaled by the AdS curvature radius $L$ (dropped below for simplicity), and the fourdimensional (4D) metric convention has been chosen as $\eta_{\mu \nu}=(+1,-1,-1,-1)$.

The action of the improved soft-wall AdS/QCD model in the two-flavor case [26] can be written as

$$
\begin{aligned}
S_{M}= & \int d^{5} x \sqrt{g} e^{-\Phi(z)} \cdot \operatorname{Tr}\left\{|D X|^{2}-m_{5}^{2}(z)|X|^{2}\right. \\
& \left.-\lambda|X|^{4}-\frac{1}{4 g_{5}^{2}}\left(F_{L}^{2}+F_{R}^{2}\right)\right\},
\end{aligned}
$$

where $D^{M} X=\partial^{M} X-i A_{L}^{M} X+i X A_{R}^{M}$ and $F_{L, R}^{M N}=\partial^{M} A_{L, R}^{N}-$ $\partial^{N} A_{L, R}^{M}-i\left[A_{L, R}^{M}, A_{L, R}^{N}\right]$, with $A_{L}^{M}=A_{L}^{a, M} t_{L}^{a}, A_{R}^{M}=A_{R}^{a, M} t_{R}^{a}$, in which $t_{L}^{a}$ and $t_{R}^{a}$ are the generators of $\mathrm{SU}(2)_{L}$ and $\mathrm{SU}(2)_{R}$. The dilaton field $\Phi(z)=\mu_{g}^{2} z^{2}$ leads to the Regge behavior of the meson spectrum [12]. The key point of this model is the introduction of a quartic term of the bulk scalar field $X(x, z)$ and a $z$-dependent bulk mass $m_{5}^{2}(z)$ of $X(x, z)$, which are closely related to the chiral symmetry breaking and thus are crucial for a consistent description of both the meson spectrum and chiral phase transition [26]. We have used the simplest form $m_{5}^{2}(z)=-3-\mu_{c}^{2} z^{2}$ in accordance with the constrained UV and IR asymptotics.

The mass spectra of $\pi, f_{0}, \rho$, and $a_{1}$ mesons have been calculated after fixing all the parameters of the model in [26]. Furthermore, this model also reproduces the same two-flavor chiral transition behavior as that shown in Fig. 1. In the chiral limit, it gives a second-order chiral phase transition, and at finite quark masses it displays a crossover behavior. Hence, it makes sense to generalize this model to include the strange quark sector.

\section{B. Generalization to the $\mathbf{2}+\mathbf{1}$-flavor case}

It has been shown that an additional term, viz., the ' $t$ Hooft determinant $\operatorname{det}[X]$, needs to be considered for the correct realization of the chiral phase transition in the flavor-symmetric case $[67,68]$. We drop the sector of chiral gauge fields in the action (2), which is irrelevant for the chiral phase transition in our consideration. Thus the action to be addressed in the $2+1$-flavor case is

$$
\begin{aligned}
S= & \int d^{5} x \sqrt{g} e^{-\Phi(z)} \cdot\left\{\operatorname{Tr}\left[|D X|^{2}-m_{5}^{2}(z)|X|^{2}-\lambda|X|^{4}\right]\right. \\
& -\gamma \operatorname{det}[X]\} .
\end{aligned}
$$

To study the chiral transition at finite temperatures in the holographic framework, we use the AdS-Schwarzschild black hole as the simplest ansatz:

$$
d s^{2}=e^{2 A(z)}\left(f(z) d t^{2}-d x^{i 2}-\frac{d z^{2}}{f(z)}\right),
$$

with

$$
f(z)=1-\frac{z^{4}}{z_{h}^{4}}
$$

where the horizon of the black hole $z_{h}$ is related to the Hawking temperature $T$ by the formula

$$
T=\frac{1}{4 \pi}\left|\frac{d f}{d z}\right|_{z_{h}}=\frac{1}{\pi z_{h}} .
$$

As the close relations with the properties of linear confinement and chiral symmetry breaking, the dilaton field $\Phi(z)$ and the bulk scalar mass $m_{5}^{2}(z)$ will take the same forms as those in the two-flavor case 


$$
\Phi(z)=\mu_{g}^{2} z^{2}, \quad m_{5}^{2}(z)=-3-\mu_{c}^{2} z^{2} .
$$

Following the previous studies in $[67,68]$, the vacuum expectation value (VEV) of the bulk scalar field $X(z)$ in the $2+1$-flavor case is assumed to be

$$
\langle X\rangle=\frac{1}{\sqrt{2}}\left(\begin{array}{ccc}
\chi_{u}(z) & 0 & 0 \\
0 & \chi_{d}(z) & 0 \\
0 & 0 & \chi_{s}(z)
\end{array}\right),
$$

with $\chi_{u}(z)=\chi_{d}(z)$. According to AdS/CFT, the chiral condensates of $u(d)$ and $s$ quarks are contained in the UV expansion of $\langle X\rangle[10,11]$; thus, to study the chiral transition behavior, we only need to consider the equation of motion of $\langle X\rangle$, which can be derived from the action (3) as

$$
\begin{aligned}
\chi_{u}^{\prime \prime} & +\left(\frac{f^{\prime}}{f}+3 A^{\prime}-\Phi^{\prime}\right) \chi_{u}^{\prime} \\
- & \frac{e^{2 A}}{f}\left(m_{5}^{2} \chi_{u}+\lambda \chi_{u}^{3}+\frac{\gamma}{2 \sqrt{2}} \chi_{u} \chi_{s}\right)=0, \\
\chi_{s}^{\prime \prime} & +\left(\frac{f^{\prime}}{f}+3 A^{\prime}-\Phi^{\prime}\right) \chi_{s}^{\prime} \\
& -\frac{e^{2 A}}{f}\left(m_{5}^{2} \chi_{s}+\lambda \chi_{s}^{3}+\frac{\gamma}{2 \sqrt{2}} \chi_{u}^{2}\right)=0 .
\end{aligned}
$$

\section{Boundary conditions}

By the prescription of AdS/CFT [10,11], the UV asymptotic forms of $\chi_{u}(z)$ and $\chi_{s}(z)$ can be directly solved from Eqs. (9) and (10):

$$
\begin{aligned}
\chi_{u}(z \sim 0)= & m_{u} \zeta z-\frac{m_{u} m_{s} \gamma \zeta^{2}}{2 \sqrt{2}} z^{2}+\frac{\sigma_{u}}{\zeta} z^{3} \\
& +\frac{1}{16} m_{u} \zeta\left(-m_{s}^{2} \gamma^{2} \zeta^{2}-m_{u}^{2} \gamma^{2} \zeta^{2}+8 m_{u}^{2} \zeta^{2} \lambda\right. \\
& \left.+16 \mu_{g}^{2}-8 \mu_{c}^{2}\right) z^{3} \ln z+\cdots, \\
\chi_{s}(z \sim 0)= & m_{s} \zeta z-\frac{m_{u}^{2} \gamma \zeta^{2}}{2 \sqrt{2}} z^{2}+\frac{\sigma_{s}}{\zeta} z^{3} \\
& +\frac{1}{8}\left(-m_{s} m_{u}^{2} \gamma^{2} \zeta^{3}+4 m_{s}^{3} \zeta^{3} \lambda\right. \\
& \left.+8 m_{s} \zeta \mu_{g}^{2}-4 m_{s} \zeta \mu_{c}^{2}\right) z^{3} \ln z+\cdots,
\end{aligned}
$$

where $m_{u}, m_{s}$ denote the quark masses and $\sigma_{u}, \sigma_{s}$ are the chiral condensates. The normalization constant $\zeta=\frac{\sqrt{N_{c}}}{2 \pi}$ is fixed by the correct $N_{c}$ scaling behavior of the quark mass and chiral condensate [19]. Note that there are only two independent parameters in the UV expansion of $\chi_{u}(z)$ or $\chi_{s}(z)$, i.e., the quark mass $m_{u, s}$ and the chiral condensate $\sigma_{u, s}$, which are most relevant to our discussion of the chiral phase transition.
As Eqs. (9) and (10) are a system of second-order nonlinear ordinary differential equations, we must impose proper boundary conditions to solve them. The first derivatives of $\chi_{u}(z)$ and $\chi_{s}(z)$ at $z=0$ will be used as the UV boundary condition in the numerical calculation

$$
\chi_{u}^{\prime}(0)=m_{u} \zeta, \quad \chi_{s}^{\prime}(0)=m_{s} \zeta .
$$

Note that Eqs. (9) and (10) are singular at the horizon of the black hole, which can be seen from the expansion of these equations at $z=z_{h}$ :

$$
\begin{aligned}
& \frac{1}{16 z_{h}\left(z-z_{h}\right)}\left\{-12 \chi_{u}\left(z_{h}\right)-4 z_{h}^{2} \mu_{c}^{2} \chi_{u}\left(z_{h}\right)\right. \\
& \left.\quad+\sqrt{2} \gamma \chi_{s}\left(z_{h}\right) \chi_{u}\left(z_{h}\right)+4 \lambda \chi_{u}^{3}\left(z_{h}\right)+16 z_{h} \chi_{u}^{\prime}\left(z_{h}\right)\right\} \\
& \quad+\mathcal{O}\left(z-z_{h}\right) \\
& \quad \frac{1}{16 z_{h}\left(z-z_{h}\right)}\left\{-12 \chi_{s}\left(z_{h}\right)-4 z_{h}^{2} \mu_{c}^{2} \chi_{s}\left(z_{h}\right)\right. \\
& \left.\quad+\sqrt{2} \gamma \chi_{u}^{2}\left(z_{h}\right)+4 \lambda \chi_{s}^{3}\left(z_{h}\right)+16 z_{h} \chi_{s}^{\prime}\left(z_{h}\right)\right\} \\
& \quad+\mathcal{O}\left(z-z_{h}\right)
\end{aligned}
$$

These singular forms at horizon $z=z_{h}$ supply us with a natural IR boundary condition to guarantee the regular near-horizon behaviors of $\chi_{u}(z)$ and $\chi_{s}(z)$, i.e.,

$$
\begin{aligned}
& -12 \chi_{u}\left(z_{h}\right)-4 z_{h}^{2} \mu_{c}^{2} \chi_{u}\left(z_{h}\right)+\sqrt{2} \gamma \chi_{s}\left(z_{h}\right) \chi_{u}\left(z_{h}\right) \\
& \quad+4 \lambda \chi_{u}^{3}\left(z_{h}\right)+16 z_{h} \chi_{u}^{\prime}\left(z_{h}\right)=0, \\
& -12 \chi_{s}\left(z_{h}\right)-4 z_{h}^{2} \mu_{c}^{2} \chi_{s}\left(z_{h}\right)+\sqrt{2} \gamma \chi_{u}^{2}\left(z_{h}\right) \\
& \quad+4 \lambda \chi_{s}^{3}\left(z_{h}\right)+16 z_{h} \chi_{s}^{\prime}\left(z_{h}\right)=0 .
\end{aligned}
$$

From Eqs. (9) and (10) and with the boundary conditions (13), (16), and (17), we can solve the VEVs of the bulk scalar field $\chi_{u}(z)$ and $\chi_{s}(z)$ numerically by the spectral collocation method $[74,75]$. For one set of quark masses $\left(m_{u}, m_{s}\right)$ and a given value of temperature, there will be one set of (or several sets of) solutions $\left(\chi_{u}, \chi_{s}\right)$ with regular IR behaviors at the black hole horizon $z_{h}$. Using the UV asymptotic expansions (11) and (12), we can extract the values of the condensates $\left(\sigma_{u}, \sigma_{s}\right)$ from the solutions $\left(\chi_{u}, \chi_{s}\right)$.

\section{CHIRAL PHASE TRANSITION IN THE 2 + 1-FLAVOR CASE}

\section{A. Parameters and the main results}

After fixing the general setup, we now study the $2+1$ flavor chiral phase transition in our model. To solve Eqs. (9) and (10), we need first to fix the four parameters $\mu_{c}, \mu_{g}, \gamma$, and $\lambda$ in the model. It should be noted that the parameters determined by the hadron spectra in the 
two-flavor case [26] have little reference value in our case here as the strange quark contributions cannot be neglected in the 2+1-flavor case. Thus, to fix the parameters of the model, we need to calculate the low-energy quantities with the ingredients related to the strange quark included, which will not be addressed in this work. Nevertheless, as pointed out in [26], the parameters $\mu_{c}$ and $\mu_{g}$ are intimately linked with the properties of chiral symmetry breaking and linear confinement. The value of $\mu_{c}$ is around the energy scale of chiral symmetry breaking $\mu_{c} \sim \Lambda_{\chi} \sim 1 \mathrm{GeV}$, while the value of $\mu_{g}$ is close to the $\Lambda_{\mathrm{QCD}}$ energy scale $\mu_{g} \sim \Lambda_{\mathrm{QCD}}$ [76]. These two parameters have intrinsic meanings and should not be changed significantly. Here we just take the same values of $\mu_{c}$ and $\mu_{g}$ as in [26], i.e., $\mu_{c}=1180 \mathrm{MeV}$ and $\mu_{g}=440 \mathrm{MeV}$. The choosing of the couplings $\gamma$ and $\lambda$ is relatively arbitrary, except that $\gamma$ must be negative to generate a first-order phase transition [68], and we take $\gamma=-22.6$ and $\lambda=16.8$ in our analysis below.

We first sketch our main results, which are summarized in the $\left(m_{u, d}, m_{s}\right)$ phase diagram in Fig. 2. A detailed analysis for different cases will be given below. Note that we do not address the pure gauge sector corresponding to the upper right corner of the phase diagram. In Fig. 2, the blue curve represents the second-order transition line, which divides the $\left(m_{u, d}, m_{s}\right)$ plane into a first-order transition region and a crossover region (note the different coordinate range of the $m_{s}$ and $m_{u, d}$ axes). We can see that the calculated phase diagram is fully consistent with the phase diagram shown in Fig. 1, at least qualitatively. We also see that the physical point is in the crossover region with the parameter values given above. The most interesting thing is that a tricritical point also appears on the $m_{u}=0$ boundary of the calculated phase diagram, which supports the standard scenario in Fig. 1.

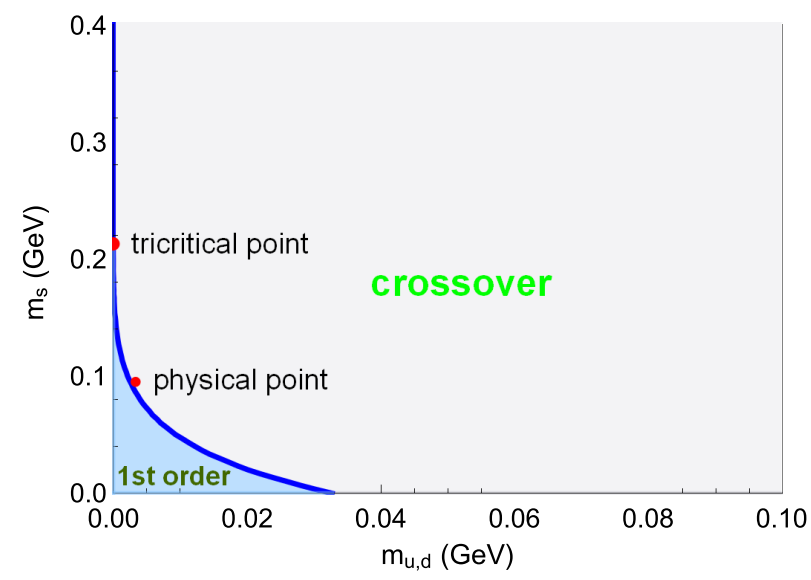

FIG. 2. The calculated $\left(m_{u, d}, m_{s}\right)$ phase diagram in the improved soft-wall model, where the parameters are taken to be $\mu_{c}=1180 \mathrm{MeV}, \mu_{g}=440 \mathrm{MeV}, \gamma=-22.6$, and $\lambda=16.8$. The blue curve denotes the second-order transition line.

\section{B. The physical point and a typical first-order transition point}

We proceed to give a detailed analysis of the calculated phase diagram in Fig. 2. According to Sec. II C, provided that the quark masses $\left(m_{u}, m_{s}\right)$ are given, we can solve the $\operatorname{VEVs}\left(\chi_{u}, \chi_{s}\right)$ from Eqs. (9) and (10) at each temperature; extract the chiral condensates $\left(\sigma_{u}, \sigma_{s}\right)$ from the UV expansion of $\left(\chi_{u}, \chi_{s}\right)$; and then obtain the chiral transition properties of $\left(\sigma_{u}, \sigma_{s}\right)$.

We present the calculated results at the physical point with $m_{u}=3.336 \mathrm{MeV}$ and $m_{s}=95 \mathrm{MeV}$ [77] in Fig. 3 . The upper panel shows the chiral transition of the condensates $\sigma_{u}, \sigma_{s}$ with temperature $T$, from which we can see the crossover behavior obviously. The lower panel shows the solutions of VEVs $\left(\chi_{u}, \chi_{s}\right)$ at three different temperatures. We can see that $\left(\chi_{u}, \chi_{s}\right)$ would approach the zero solution $(0,0)$ with the increase of temperature $T$, which is easy to understand. As the temperature characterizes the energy scale, the physical quark masses can be neglected at very high temperatures, in which case Eqs. (9) and (10) have a null solution $\left(\chi_{u}, \chi_{s}\right)=(0,0)$. From the upper panel of Fig. 3 we also see that at low temperatures the condensate $\sigma_{s}$ is suppressed compared with the condensate
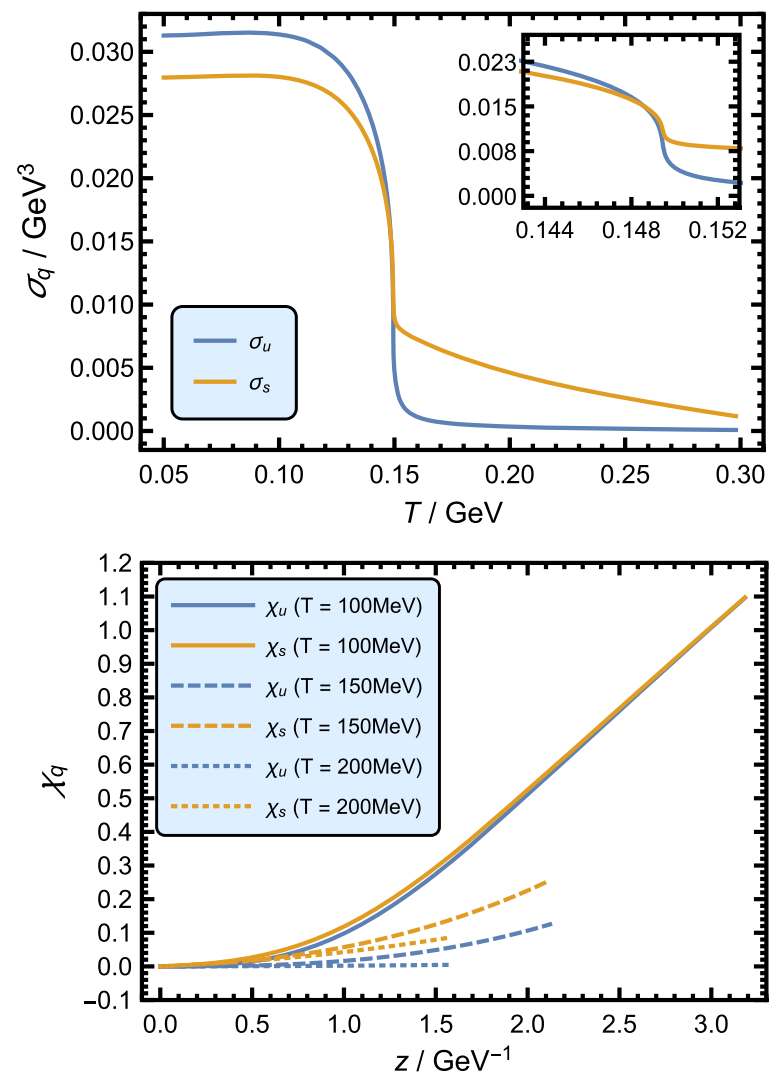

FIG. 3. Upper panel: The chiral transition behavior of $\sigma_{u}$ and $\sigma_{s}$ with temperature $T$ at the physical point $m_{u}=3.336 \mathrm{MeV}$, $m_{s}=95 \mathrm{MeV}$. Lower panel: The solutions of VEVs $\left(\chi_{u}, \chi_{s}\right)$ at temperatures $T=100,150,200 \mathrm{MeV}$. 

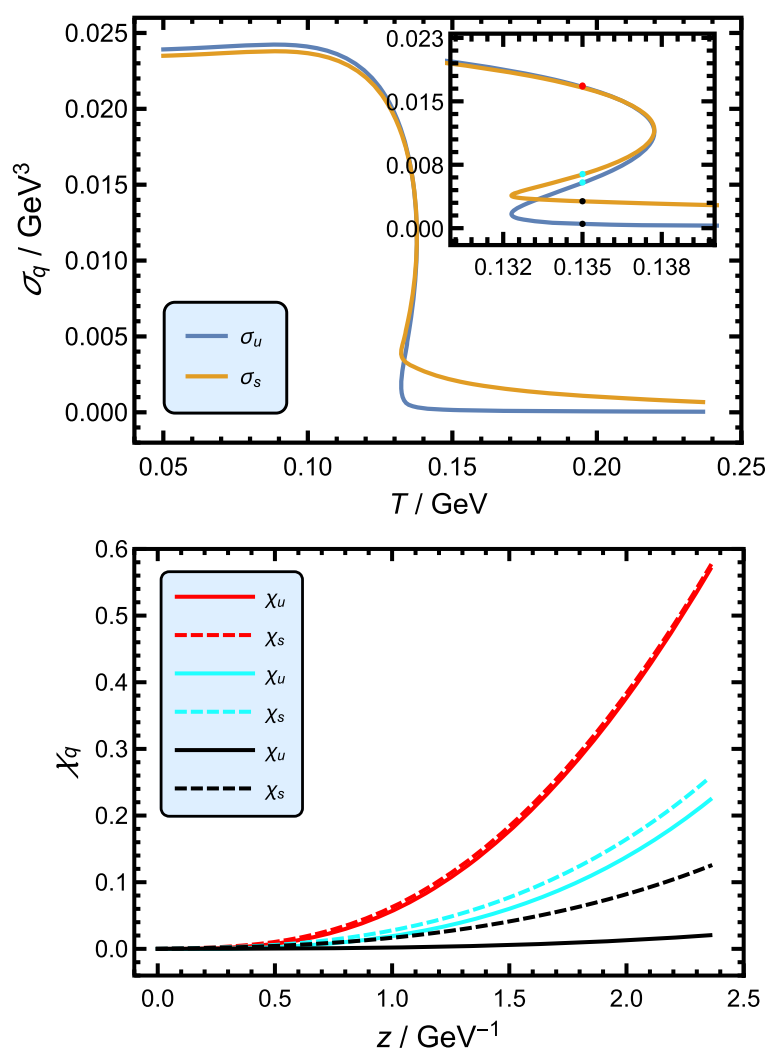

FIG. 4. Upper panel: The chiral transition behavior of $\sigma_{u}$ and $\sigma_{s}$ with temperature $T$ at the point $\left(m_{u}, m_{s}\right)=(1 \mathrm{MeV}, 20 \mathrm{MeV})$. Lower panel: The three solutions of the VEVs $\left(\chi_{u}, \chi_{s}\right)$ at $T=135 \mathrm{MeV}$, where the corresponding condensates $\left(\sigma_{u}, \sigma_{s}\right)$ in the upper panel (see the insert) have been dotted with the same color as that of the solutions in the lower panel.

$\sigma_{u}$; however, after crossing the pseudocritical transition region around $T \simeq 0.15 \mathrm{GeV}, \sigma_{u}$ drops more quickly than $\sigma_{s}$ and becomes smaller than $\sigma_{s}$ at larger temperatures.

In Fig. 4, we also display a typical first-order phase transition at the point $\left(m_{u}, m_{s}\right)=(1 \mathrm{MeV}, 20 \mathrm{MeV})$. The upper panel shows the chiral transition behavior of the condensates $\left(\sigma_{u}, \sigma_{s}\right)$ with the temperature $T$, from which we can see an inflection in the transition region with a sudden decrease of the condensate (see the inset), which indicates a first-order phase transition. Note that Eqs. (9) and (10) have three solutions at each temperature of the inflection region. We show the three solutions at temperature $T=135 \mathrm{MeV}$ in the lower panel of Fig. 4. In principle, we can calculate the free energies corresponding to these solutions to quantify the first-order transition and to fix the critical temperature, as has been done in [68]. Here it is enough for us to know the order of phase transitions.

\section{The flavor-symmetric case}

In the flavor-symmetric case $\left(m_{u}=m_{d}=m_{s}\right)$, there is no distinction between the VEVs $\chi_{u}$ and $\chi_{s}$. Thus, Eqs. (9) and (10) will reduce to one equation, i.e.,

$$
\begin{aligned}
\chi^{\prime \prime} & +\left(\frac{f^{\prime}}{f}+3 A^{\prime}-\Phi^{\prime}\right) \chi^{\prime} \\
& -\frac{e^{2 A}}{f}\left(m_{5}^{2} \chi+\lambda \chi^{3}+\frac{\gamma}{2 \sqrt{2}} \chi^{2}\right)=0,
\end{aligned}
$$

which has been considered in the previous works (see, e.g., $[67,68])$. We can also deal with the two coupled equations (9) and (10) directly and the result will be the same as that solved from the single equation.

We present the calculated results of the improved softwall AdS/QCD model in Fig. 5, where the chiral transition behavior of $\left(\sigma_{u}, \sigma_{s}\right)$ with four different quark masses is plotted. The first two panels show obvious inflections in the transition region, which implies a first-order phase transition. The third panel shows a near second-order phase transition, while the fourth one is a crossover transition. We see that at small quark masses the chiral transition is first order and becomes a second-order one when the quark mass increases up to a point and then makes a crossover transition, which is consistent with the phase diagram in Fig. 1. We can also see roughly from Fig. 5 that the (pseudo)critical transition temperature increases with increasing quark mass, at least in a finite range of quark masses, which agrees with the lattice result [6].

\section{The $\boldsymbol{m}_{s}=0$ case}

In the $m_{s}=0$ case, we need to solve the two coupled equations (9) and (10) to obtain the set of solutions $\left(\chi_{u}, \chi_{s}\right)$, from which the condensates $\left(\sigma_{u}, \sigma_{s}\right)$ can be extracted. We plot the chiral transitions of $\left(\sigma_{u}, \sigma_{s}\right)$ with temperature $T$ for the four selected $u$ quark masses in Fig. 6, from which we can see that with the increase of the quark mass $m_{u}$ a similar transition behavior happens as that in the flavor-symmetric case. When $m_{u}$ is small (see the first two panels), both $\sigma_{u}$ and $\sigma_{s}$ show a first-order phase transition with an inflection appearing in the critical transition region, and then both of them become a second-order transition as $m_{u}$ reaches an end point around $32.7 \mathrm{MeV}$ (see the third panel). The chiral transitions of $\left(\sigma_{u}, \sigma_{s}\right)$ become crossover transitions when $m_{u}$ is larger than the value of the second-order transition point (see the fourth panel).

Contrary to the case of the physical point in Fig. 3, the condensate $\sigma_{s}$ is larger than $\sigma_{u}$ at low temperatures and smaller than $\sigma_{u}$ at high temperatures in the $m_{s}=0$ case. We find that this is always the case in the lower right region of our calculated phase diagram, with $m_{u, d}=m_{s}$ as the dividing line, as can be expected. In the upper left region, the relationship between $\sigma_{u}$ and $\sigma_{s}$ is reversed, just like the case of the physical point; i.e., $\sigma_{s}$ is smaller than $\sigma_{u}$ at low temperatures and larger than $\sigma_{u}$ at high temperatures. On the dividing line $m_{u, d}=m_{s}$ which corresponds to the flavor-symmetric case, $\sigma_{u}(T)$ and $\sigma_{s}(T)$ merge into a single curve for any quark mass, as shown in Fig. 5. 

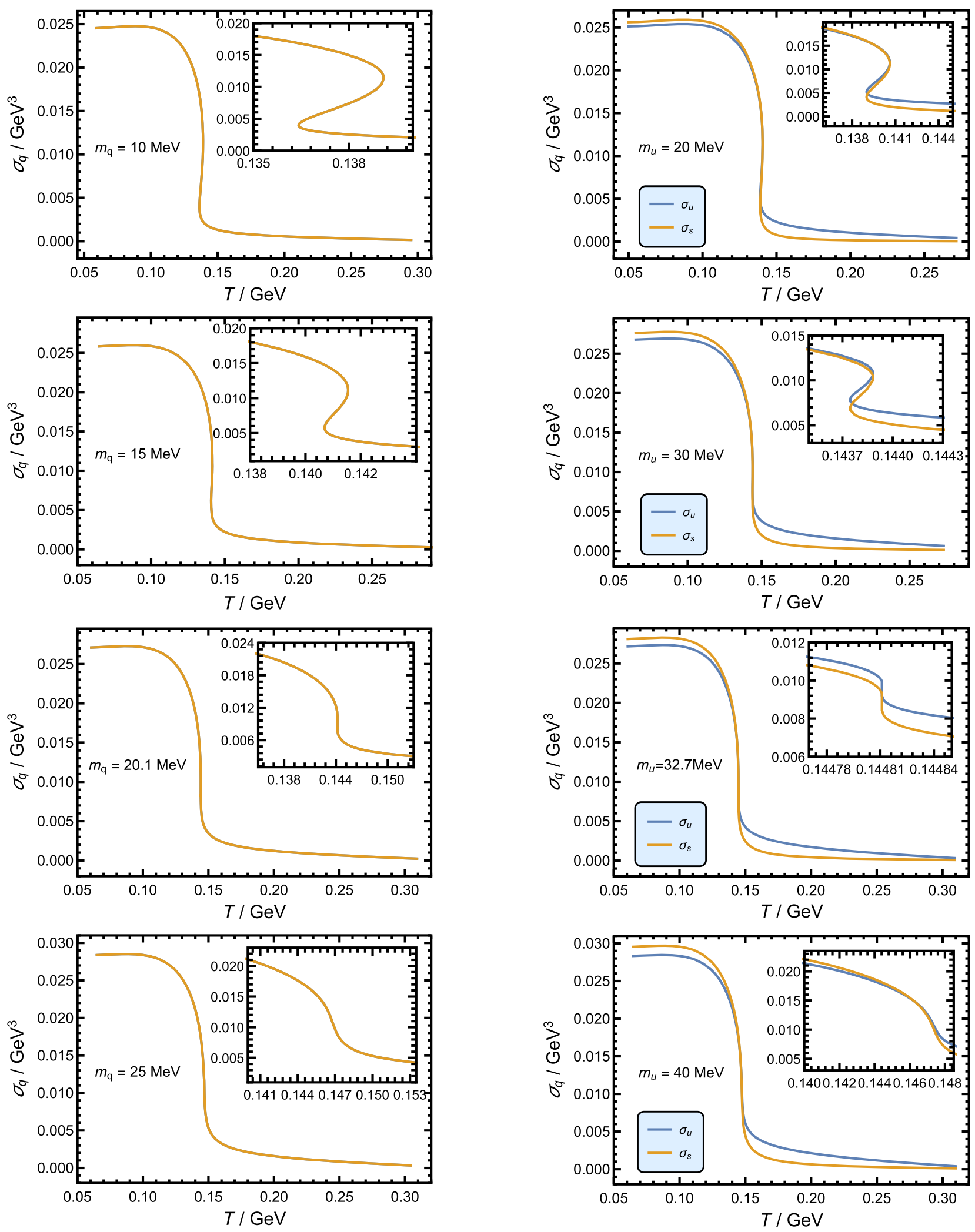

FIG. 5. The chiral transition behavior of the condensate $\left(\sigma_{q} \equiv \sigma_{u}=\sigma_{s}\right)$ with temperature $T$ at $m_{q}=10,15,20.1$, $25 \mathrm{MeV}$ in the flavor-symmetric case $\left(m_{q} \equiv m_{u}=m_{d}=m_{s}\right)$.

FIG. 6. The chiral transition behavior of the condensates $\left(\sigma_{u}, \sigma_{s}\right)$ with temperature $T$ at $m_{u}=20,30,32.7,40 \mathrm{MeV}$ in the $m_{s}=0$ case. 


\section{E. The $m_{u}=0$ case}

There are still debates on the order of the QCD phase transition for the case of small $m_{u, d}$ but large $m_{s}[6,78]$. The nature of the chiral phase transition was first discussed in [79], where it was argued that the chiral transition should be first order for three light quark flavors, while for two light quark flavors it could be either second order or first order, which is determined by to what extent the broken axial symmetry $U_{A}(1)$ is restored at the transition temperature. If the $U_{A}(1)$ symmetry is not restored at the transition temperature, the chiral phase transition in the chiral limit is most likely a second-order one belonging to the $O(4)$ universality class, which is supported by lattice simulations in recent years [80]. Indeed, the second-order phase transition already happens at some finite $m_{s}$ (tricritical point) on the $m_{u}=0$ boundary.

To obtain the chiral transition behavior in the $m_{u}=0$ case, we need to be careful to solve Eqs. (9) and (10). First note that in this case we have two sets of solutions which correspond to $\chi_{u}=0$ and $\chi_{u} \neq 0$, respectively. When $\chi_{u}=0$, Eqs. (9) and (10) reduce to one equation of $\chi_{s}$,

$\chi_{s}^{\prime \prime}+\left(\frac{f^{\prime}}{f}+3 A^{\prime}-\Phi^{\prime}\right) \chi_{s}^{\prime}-\frac{e^{2 A}}{f}\left(m_{5}^{2} \chi_{s}+\lambda \chi_{s}^{3}\right)=0$,

whose solution will be labeled as $\chi_{s}^{0}$. Thus we have a set of solutions for Eqs. (9) and (10), viz., $\left(\chi_{u}, \chi_{s}\right)=\left(0, \chi_{s}^{0}\right)$. For another set of solutions $\left(\chi_{u}, \chi_{s}\right)$ with $\chi_{u} \neq 0$, we need to solve the two coupled equations (9) and (10) directly.

The numerical results of the chiral transition behavior of $\sigma_{u}$ and $\sigma_{s}$ are shown in Fig. 7, where we also choose four typical $s$ quark masses for our consideration. It should be noted that both $\sigma_{u}(T)$ and $\sigma_{s}(T)$ in Fig. 7 are composed of two parts, of which one corresponds to the solution $\left(0, \chi_{s}^{0}\right)$ at higher temperatures and the other part corresponds to the solution $\left(\chi_{u} \neq 0, \chi_{s}\right)$ at lower temperatures. We also note that Eqs. (9) and (10) have no solution $\left(\chi_{u} \neq 0, \chi_{s}\right)$ at higher temperatures, and we have dropped the low-temperature part of the solution $\left(0, \chi_{s}^{0}\right)$ in Fig. 7 by the stability consideration [68]. As $\sigma_{u}(T)$ and $\sigma_{s}(T)$ come from two sets of solutions of Eqs. (9) and (10), there must be no crossover transition in the $m_{u}=0$ case. From the upper two panels of Fig. 7, we can see obviously the feature of inflection around the transition region, which implies a first-order phase transition at $m_{s}=100,150 \mathrm{MeV}$. When $m_{s}$ is large enough, the inflection disappears, which signifies a second-order phase transition (see the lower two panels). The tricritical point, viz., the separation between the first-order and second-order phase transitions on the $m_{u}=0$ boundary, is around $m_{s} \simeq 212.7 \mathrm{MeV}$. Thus the results obtained from the improved soft-wall AdS/QCD model support the
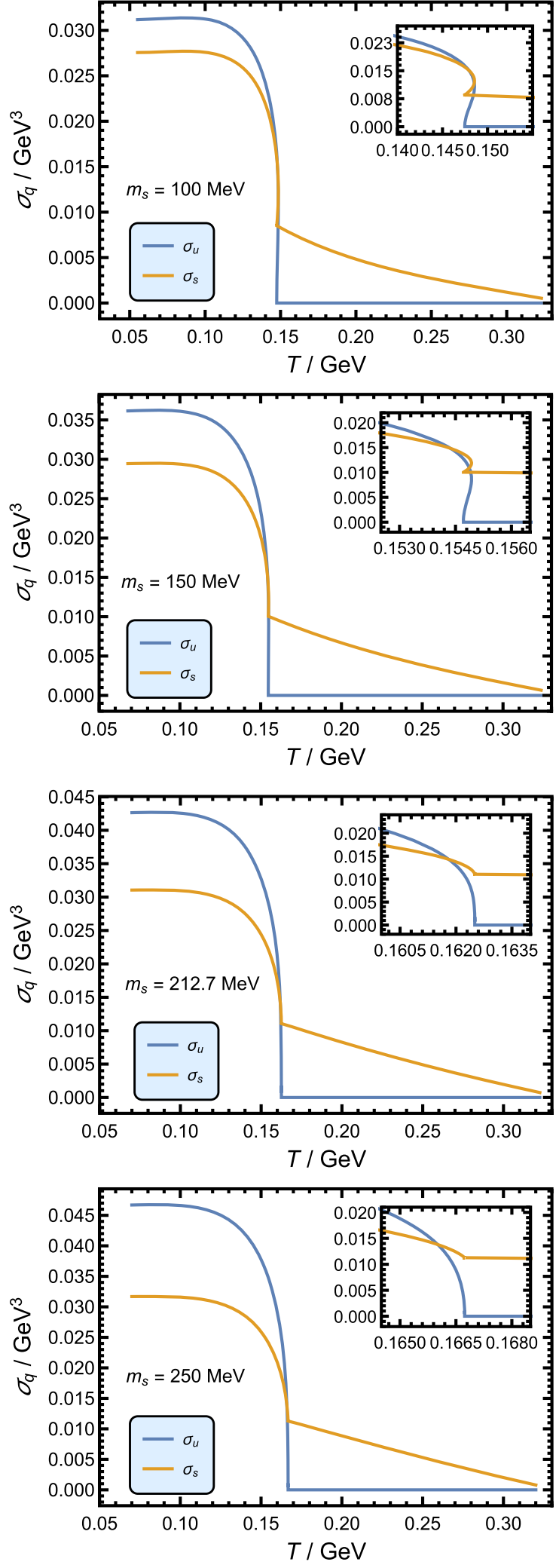

FIG. 7. The chiral transition behavior of the condensates $\left(\sigma_{u}, \sigma_{s}\right)$ with the temperature $T$ at $m_{s}=100,150,212.7,250 \mathrm{MeV}$ in the $m_{u, d}=0$ case. 
standard scenario of the $\left(m_{u, d}, m_{s}\right)$ phase diagram in Fig. 1.

\section{IV. $\gamma$ DEPENDENCE OF THE PHASE DIAGRAM}

As mentioned in Sec. III A, the couplings $\gamma$ and $\lambda$ of the improved soft-wall AdS/QCD model are more or less arbitrary in our discussion of the chiral phase transition. In this section, we consider the effects of $\gamma$ on the chiral transition behavior and the $\left(m_{u, d}, m_{s}\right)$ phase diagram with the coupling $\lambda=16.8$ fixed as before. We choose two other values of $\gamma$ besides the one used in the preceding sections, i.e., $\gamma=-19,-22.6,-24.4$. The second-order transition lines of the phase diagram for the three different values of $\gamma$ are shown in the upper panel of Fig. 8, from which we can see that the second-order line moves upward as $\gamma$ decreases (note that $\gamma \leq 0$ ). In other words, the region of the firstorder phase transition would shrink with the increase of $\gamma$.

In the lower panel of Fig. 8, we plot the chiral transition behavior of the condensate at $m_{u, d}=m_{s}=0$ when $\gamma$ takes the above three values and also $\gamma=0$. The first-order transition behavior of the condensate is obvious when $\gamma$ takes negative values. However, it becomes a second-order
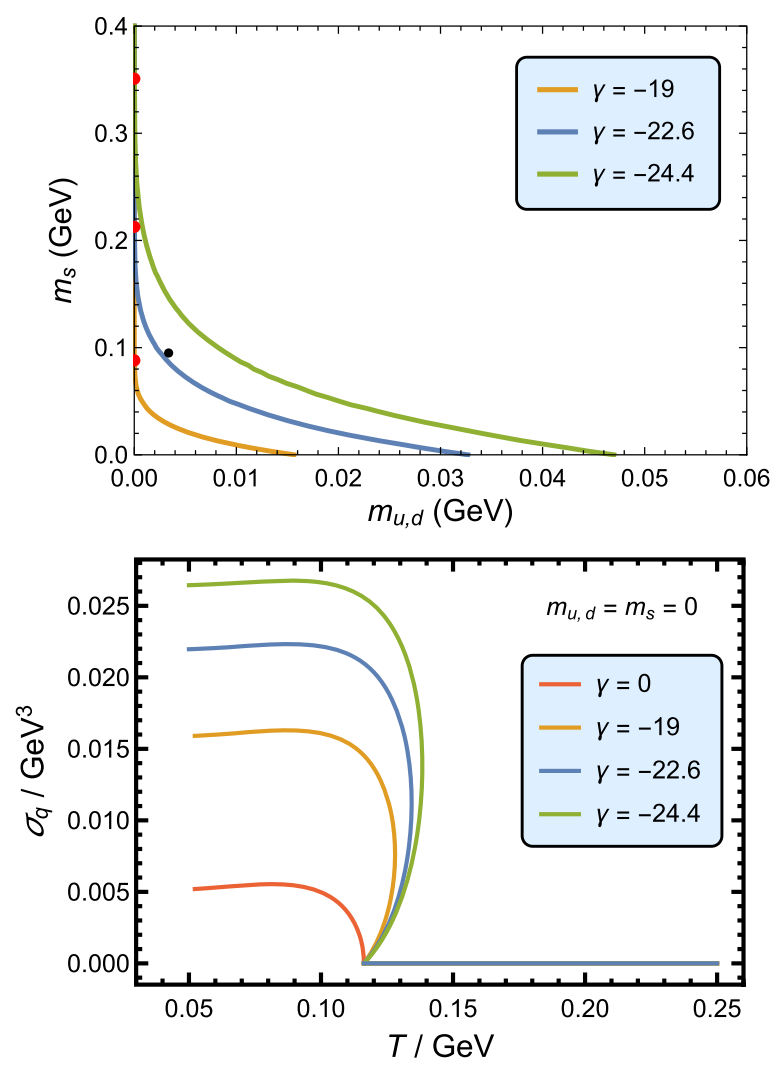

FIG. 8. Upper panel: The calculated $\left(m_{u, d}, m_{s}\right)$ phase diagrams in the improved soft-wall model with different values of $\gamma$, where the red dot denotes the tricritical point and the black dot denotes the physical point. Lower panel: The chiral transition behavior of the condensate with temperature $T$ at $m_{u, d}=m_{s}=0$ for different values of $\gamma$. phase transition when $\gamma=0$, which indicates that the region of the first-order transition reduces to zero. This can be seen directly from Eqs. (9) and (10), which will decouple and reduce to a single equation similar as that in the two-flavor case when the determinant term vanishes $(\gamma=0)$. From the lower panel of Fig. 8, we can also see that the condensate at zero or low temperatures will decrease with the increase of $\gamma$.

\section{CONCLUSION AND DISCUSSION}

In this work, by adding the 't Hooft determinant term $\operatorname{det}[X]$, we generalized the improved soft-wall AdS/QCD model from the two-flavor case [26] to the $2+1$-flavor case. We then gave a detailed analysis of the chiral transition behavior at different quark masses and obtained the $\left(m_{u, d}, m_{s}\right)$ phase diagram for the quark sector (see Fig. 2). Quite strikingly, the calculated phase diagram in a simple AdS/QCD model is completely consistent with the standard scenario shown in Fig. 1. It is interesting to find that a tricritical point also exists on the $m_{u}=0$ boundary in the holographic calculation, which is supported by the lattice simulations [80]. This tricritical point separates the lower first-order transition region from the upper $O(4)$ second-order line at $m_{u}=0$, which implies that in the chiral limit of a two-flavor case the chiral phase transition is second order (consistent with our previous result in [26]). However, the reason for the $O(4)$ and $Z(2)$ nature of the second-order line separated by the tricritical point is not clear in our analysis.

In the improved soft-wall AdS/QCD model, there are four parameters relevant to our discussion of chiral phase transition, viz., $\mu_{c}, \mu_{g}, \gamma$, and $\lambda$. As the parameters $\mu_{c}$ and $\mu_{g}$ are related to the chiral symmetry breaking and $\Lambda_{\mathrm{QCD}}$ energy scales, respectively, there are only two relatively arbitrary parameters $\gamma$ and $\lambda$ which might be fixed by the hadron spectrum with $2+1$ quark flavors. With the parameter $\lambda$ fixed, we studied the effects of the parameter $\gamma$ on the $2+1$ chiral transition and the $\left(m_{u, d}, m_{s}\right)$ phase diagram. We find that the first-order transition region shrinks with the increase of $\gamma(\gamma \leq 0)$ and finally disappears as $\gamma$ vanishes.

In spite of the good descriptions for the chiral phase transition and the low-energy hadron physics such as the meson spectrum in the improved soft-wall AdS/QCD model, several limitations of our work should be pointed out here. Following many other researches (see, e.g., [47-51]), to study the temperature dependence of a chiral condensate, we only used the AdS black hole solution, which yet characterizes the deconfined phase according to the arguments in $[43,81]$, while the low-temperature confined phase is characterized by a thermal AdS solution. The gravity dual of the transition between these two phases is a first-order Hawking-Page type, which is consistent with the analysis from pure Yang-Mills theories. However, the question of how to realize the smooth crossover behavior of 
quark matters is not very clear in this prescription, let alone the whole phase diagram in the quark-mass plane. As a phenomenological study, we may hope that the black hole solution would still be valid for the description of chiral transitions in the region slightly below the critical temperature [47,50], and the realization of both the meson spectrum and the chiral phase structures in a simple soft-wall model may offer useful guidance in the further development of holographic QCD.

As a first try, the backreaction of the quark sectors to the thermal background is not included in this work, which needs to be considered in further study, and many other works might deserve to be done in this framework. For instance, as we have said, the hadron spectrum with $2+1$ quark flavors can be used to fix the parameters in this model. However, this entails a considerable extension of the model to include the strange quark sector [24]. We can also study the chemical potential effects and the $\mu-T$ phase diagram by introducing a $U(1)$ gauge field in the model. To characterize the QCD phase transition more completely, we should investigate the equation of states and the relevant QCD thermodynamics, which are intimately associated with the color deconfining process. The expectation value of the Polyakov loop $\langle L\rangle$ can also be calculated for the deconfining phase transition.

\section{ACKNOWLEDGMENTS}

The authors are grateful to Y. Tian for valuable discussions on the numerical method and would also like to thank D. N. Li for the discussions in the early stage of this work. This work was supported by National Science Foundation of China (NSFC) (Grants No. 11690022, No. 11475237, and No. 11121064) and the Strategic Priority Research Program of the Chinese Academy of Sciences (Grant No. XDB23030100), as well as the CAS Center for Excellence in Particle Physics (CCEPP), and is also partially supported by the China Postdoctoral Science Foundation (Grant No. 2016M601106).

\section{APPENDIX: IMPLEMENTATION OF SPECTRAL COLLOCATION METHOD IN OUR MODEL}

We now detail the implementation of the spectral collocation method in the calculation of the chiral phase transition. First we change the form of Eqs. (9) and (10) by applying the following two transformations successively:

$$
\begin{aligned}
z \rightarrow u z_{h}, & \quad \chi_{u}(z) \rightarrow \hat{\chi}_{u}(u), \quad \chi_{s}(z) \rightarrow \hat{\chi}_{s}(u), \\
u & \rightarrow \frac{t+1}{2}, \quad \hat{\chi}_{u}(u) \rightarrow \frac{t+1}{2} \tilde{\chi}_{u}(t), \\
\hat{\chi}_{s}(u) & \rightarrow \frac{t+1}{2} \tilde{\chi}_{s}(t) .
\end{aligned}
$$

Thus Eqs. (9) and (10) are transformed into

$$
\begin{aligned}
& p_{1}(t) \tilde{\chi}_{u}^{\prime \prime}(t)+p_{2}(t) \tilde{\chi}_{u}^{\prime}(t)+p_{3}(t) \tilde{\chi}_{u}(t) \\
& \quad+p_{4}(t) \tilde{\chi}_{u}(t) \tilde{\chi}_{s}(t)+p_{5}(t) \tilde{\chi}_{u}(t)^{3}=0, \\
& p_{1}(t) \tilde{\chi}_{s}^{\prime \prime}(t)+p_{2}(t) \tilde{\chi}_{s}^{\prime}(t)+p_{3}(t) \tilde{\chi}_{s}(t) \\
& \quad+p_{4}(t) \tilde{\chi}_{u}(t)^{2}+p_{5}(t) \tilde{\chi}_{s}(t)^{3}=0,
\end{aligned}
$$

where $t \in(-1,1)$, and the coefficient functions are

$$
\begin{aligned}
p_{1}(t)= & -2\left(-15-11 t+10 t^{2}+10 t^{3}+5 t^{4}+t^{5}\right), \\
p_{2}(t)= & -38-15 z_{h}^{2} \mu_{g}^{2}+6 t^{5} z_{h}^{2} \mu_{g}^{2}+t^{6} z_{h}^{2} \mu_{g}^{2} \\
& -t^{2}\left(36+z_{h}^{2} \mu_{g}^{2}\right)+4 t^{3}\left(-6+5 z_{h}^{2} \mu_{g}^{2}\right) \\
& +3 t^{4}\left(-2+5 z_{h}^{2} \mu_{g}^{2}\right)-2 t\left(12+13 z_{h}^{2} \mu_{g}^{2}\right), \\
p_{3}(t)= & (1+t)\left(-2+4 t^{3} z_{h}^{2} \mu_{g}^{2}+t^{4} z_{h}^{2} \mu_{g}^{2}+z_{h}^{2}\left(8 \mu_{c}^{2}-15 \mu_{g}^{2}\right)\right. \\
& \left.+4 t\left(-1+z_{h}^{2} \mu_{g}^{2}\right)+t^{2}\left(-2+6 z_{h}^{2} \mu_{g}^{2}\right)\right), \\
p_{4}(t)= & -4 \sqrt{2} \gamma, \\
p_{5}(t)= & -8(1+t) \lambda .
\end{aligned}
$$

The UV boundary conditions (13) now become

$$
\begin{aligned}
\tilde{\chi}_{u}(-1)-m_{u} z_{h} \zeta & =0, \\
\tilde{\chi}_{s}(-1)-m_{s} z_{h} \zeta & =0 .
\end{aligned}
$$

As mentioned in Sec. II C, the equation of motions of the improved soft-wall AdS/QCD model have natural IR boundary conditions, which will be satisfied implicitly in the following procedure.

Now we turn back to the spectral collocation method. Let us first select a set of distinct collocation points $\left\{t_{i}\right\}_{i=0}^{N}$ ( $N$ is an integer and $N \geq 1$ ) on $[-1,1]$ with $\left\{t_{i}\right\}_{i=0}^{N}$ being the $N+1$ roots of the equation,

$$
\left(1-t^{2}\right) \frac{\mathrm{d}}{\mathrm{d} t} L_{N}(t)=0,
$$

where $L_{N}(t)$ is the $N$ th Legendre polynomial. The derivative matrix $\mathbf{D}$ is an $(N+1) \times(N+1)$ matrix, which is given by

$$
\mathbf{D}_{i j}= \begin{cases}-\frac{N(N+1)}{4}, & i=j=0, \\ 0, & 1 \leq i=j \leq N-1, \\ \frac{N(N+1)}{4}, & i=j=N, \\ \frac{L_{N}\left(x_{i}\right)}{\left(x_{i}-x_{j}\right) L_{N}\left(x_{j}\right)}, & i \neq j .\end{cases}
$$

Note that the range of each index of the matrix $\mathbf{D}$ is $[0, N]$. For a general function $f(t), f\left(t_{i}\right)$ denotes the value of $f(t)$ at the collocation point $t_{i}$. The value of the derivative function $f^{\prime}(t)$ can be calculated by the differential matrix:

$$
\overrightarrow{\mathbf{f}^{\prime}(\mathbf{t})}=\mathbf{D} \overrightarrow{\mathbf{f}(\mathbf{t})},
$$


where

$$
\overrightarrow{\mathbf{f}(\mathbf{t})}=\left(\begin{array}{c}
f\left(t_{0}\right) \\
f\left(t_{1}\right) \\
\vdots \\
f\left(t_{N}\right)
\end{array}\right), \quad \overrightarrow{\mathbf{f}^{\prime}(\mathbf{t})}=\left(\begin{array}{c}
f^{\prime}\left(t_{0}\right) \\
f^{\prime}\left(t_{1}\right) \\
\vdots \\
f^{\prime}\left(t_{N}\right)
\end{array}\right) .
$$

The matrix of the second-order differential operator is defined as $\mathbf{D}_{2}=\mathbf{D}^{2}$.

Then we can build the discretization scheme of Eqs. (A3) and (A4):

$$
\begin{aligned}
& p_{1}\left(t_{i}\right) \sum_{j=0}^{N}\left(\mathbf{D}_{2}\right)_{i j} \tilde{\chi}_{u}\left(t_{j}\right)+p_{2}\left(t_{i}\right) \sum_{j=0}^{N} \mathbf{D}_{i j} \tilde{\chi}_{u}\left(t_{j}\right) \\
& \quad+p_{3}\left(t_{i}\right) \tilde{\chi}_{u}\left(t_{i}\right)+p_{4}\left(t_{i}\right) \tilde{\chi}_{u}\left(t_{i}\right) \tilde{\chi}_{s}\left(t_{i}\right) \\
& \quad+p_{5}\left(t_{i}\right) \tilde{\chi}_{u}\left(t_{i}\right)^{3}=0, \\
& 1 \leq i \leq N,
\end{aligned}
$$

$$
\begin{aligned}
& p_{1}\left(t_{i}\right) \sum_{j=0}^{N}\left(\mathbf{D}_{2}\right)_{i j} \tilde{\chi}_{s}\left(t_{j}\right)+p_{2}\left(t_{i}\right) \sum_{j=0}^{N} \mathbf{D}_{i j} \tilde{\chi}_{s}\left(t_{j}\right) \\
& \quad+p_{3}\left(t_{i}\right) \tilde{\chi}_{s}\left(t_{i}\right)+p_{4}\left(t_{i}\right) \tilde{\chi}_{u}\left(t_{i}\right)^{2}+p_{5}\left(t_{i}\right) \tilde{\chi}_{s}\left(t_{i}\right)^{3}=0, \\
& 1 \leq i \leq N .
\end{aligned}
$$

Because the domain of Eqs. (A3) and (A4) is $(-1,1)$, the range of the index $i$ in Eqs. (A11) and (A12) should be $[1, N-1]$. However, as the IR boundary conditions are natural boundary conditions which are satisfied implicitly in Eqs. (A11) and (A12), thus we take $1 \leq i \leq N$ with $N$ included. The UV boundary conditions (A6) now turn into

$$
\tilde{\chi}_{u}\left(t_{0}\right)-m_{u} z_{h} \zeta=0, \quad \tilde{\chi}_{s}\left(t_{0}\right)-m_{s} z_{h} \zeta=0
$$

Putting together Eqs. (A11)-(A13), we have in total $2(N+1)$ equations which can be solved numerically to obtain the values of $\left(\tilde{\chi}_{u}\left(t_{i}\right), \tilde{\chi}_{s}\left(t_{i}\right)\right)(0 \leq i \leq N)$.
[1] Y. Aoki, G. Endrodi, Z. Fodor, S. D. Katz, and K. K. Szabo, Nature (London) 443, 675 (2006).

[2] D. J. Gross, R. D. Pisarski, and L. G. Yaffe, Rev. Mod. Phys. 53, 43 (1981).

[3] Y. Nambu and G. Jona-Lasinio, Phys. Rev. 122, 345 (1961).

[4] L. D. McLerran and B. Svetitsky, Phys. Rev. D 24, 450 (1981).

[5] A. M. Polyakov, Phys. Lett. 72B, 477 (1978).

[6] E. Laermann and O. Philipsen, Annu. Rev. Nucl. Part. Sci. 53, 163 (2003).

[7] J. M. Maldacena, Adv. Theor. Math. Phys. 2, 231 (1998).

[8] S. S. Gubser, I. R. Klebanov, and A. M. Polyakov, Phys. Lett. B 428, 105 (1998).

[9] E. Witten, Adv. Theor. Math. Phys. 2, 253 (1998).

[10] L. Da Rold and A. Pomarol, Nucl. Phys. B721, 79 (2005).

[11] J. Erlich, E. Katz, D. T. Son, and M. A. Stephanov, Phys. Rev. Lett. 95, 261602 (2005).

[12] A. Karch, E. Katz, D. T. Son, and M. A. Stephanov, Phys. Rev. D 74, 015005 (2006).

[13] G. F. de Teramond and S. J. Brodsky, Phys. Rev. Lett. 94, 201601 (2005).

[14] J. Babington, J. Erdmenger, N. J. Evans, Z. Guralnik, and I. Kirsch, Phys. Rev. D 69, 066007 (2004).

[15] M. Kruczenski, D. Mateos, R. C. Myers, and D. J. Winters, J. High Energy Phys. 05 (2004) 041.

[16] T. Sakai and S. Sugimoto, Prog. Theor. Phys. 113, 843 (2005).

[17] T. Sakai and S. Sugimoto, Prog. Theor. Phys. 114, 1083 (2005).

[18] C. Csaki and M. Reece, J. High Energy Phys. 05 (2007) 062.
[19] A. Cherman, T. D. Cohen, and E. S. Werbos, Phys. Rev. C 79, 045203 (2009).

[20] S. He, M. Huang, Q. S. Yan, and Y. Yang, Eur. Phys. J. C 66, 187 (2010).

[21] T. Gherghetta, J. I. Kapusta, and T. M. Kelley, Phys. Rev. D 79, 076003 (2009).

[22] T. M. Kelley, S. P. Bartz, and J. I. Kapusta, Phys. Rev. D 83, 016002 (2011).

[23] Y. Q. Sui, Y. L. Wu, Z. F. Xie, and Y. B. Yang, Phys. Rev. D 81, 014024 (2010).

[24] Y. Q. Sui, Y. L. Wu, and Y. B. Yang, Phys. Rev. D 83, 065030 (2011).

[25] L. X. Cui, Z. Fang, and Y. L. Wu, Eur. Phys. J. C 76, 22 (2016).

[26] Z. Fang, Y. L. Wu, and L. Zhang, Phys. Lett. B 762, 86 (2016).

[27] D. Li, M. Huang, and Q. S. Yan, Eur. Phys. J. C 73, 2615 (2013).

[28] D. Li and M. Huang, J. High Energy Phys. 11 (2013) 088.

[29] E. V. Shuryak, Nucl. Phys. A750, 64 (2005).

[30] G. Policastro, D. T. Son, and A. O. Starinets, Phys. Rev. Lett. 87, 081601 (2001).

[31] R.-G. Cai, Z.-Y. Nie, N. Ohta, and Y.-W. Sun, Phys. Rev. D 79, 066004 (2009).

[32] R.-G. Cai, Z.-Y. Nie, and Y.-W. Sun, Phys. Rev. D 78, 126007 (2008).

[33] C. P. Herzog, A. Karch, P. Kovtun, C. Kozcaz, and L. G. Yaffe, J. High Energy Phys. 07 (2006) 013.

[34] S. S. Gubser, Phys. Rev. D 74, 126005 (2006).

[35] R. Casero, E. Kiritsis, and A. Paredes, Nucl. Phys. B787, 98 (2007). 
[36] I. Iatrakis, E. Kiritsis, and A. Paredes, Phys. Rev. D 81, 115004 (2010).

[37] I. Iatrakis, E. Kiritsis, and A. Paredes, J. High Energy Phys. 11 (2010) 123.

[38] M. Jarvinen and E. Kiritsis, J. High Energy Phys. 03 (2012) 002.

[39] D. Li, J. Liao, and M. Huang, Phys. Rev. D 89, 126006 (2014).

[40] D. Li, S. He, and M. Huang, J. High Energy Phys. 06 (2015) 046.

[41] Z. Fang, Phys. Lett. B 758, 1 (2016).

[42] A. Ballon-Bayona, M. Ihl, J. P. Shock, and D. Zoakos, J. High Energy Phys. 10 (2017) 038.

[43] C. P. Herzog, Phys. Rev. Lett. 98, 091601 (2007).

[44] C. A. Ballon-Bayona, H. Boschi-Filho, N. R. F. Braga, and L. A. Pando Zayas, Phys. Rev. D 77, 046002 (2008).

[45] R. G. Cai and J. P. Shock, J. High Energy Phys. 08 (2007) 095.

[46] Y. Kim, B. H. Lee, S. Nam, C. Park, and S. J. Sin, Phys. Rev. D 76, 086003 (2007).

[47] O. Andreev, Phys. Rev. Lett. 102, 212001 (2009).

[48] P. Colangelo, F. Giannuzzi, and S. Nicotri, Phys. Rev. D 83, 035015 (2011).

[49] S. S. Gubser, A. Nellore, S.S. Pufu, and F. D. Rocha, Phys. Rev. Lett. 101, 131601 (2008).

[50] S. S. Gubser and A. Nellore, Phys. Rev. D 78, 086007 (2008).

[51] S. S. Gubser, S. S. Pufu, and F. D. Rocha, J. High Energy Phys. 08 (2008) 085.

[52] U. Gursoy, E. Kiritsis, L. Mazzanti, and F. Nitti, Phys. Rev. Lett. 101, 181601 (2008).

[53] U. Gursoy and E. Kiritsis, J. High Energy Phys. 02 (2008) 032.

[54] U. Gursoy, E. Kiritsis, and F. Nitti, J. High Energy Phys. 02 (2008) 019.

[55] U. Gursoy, E. Kiritsis, L. Mazzanti, and F. Nitti, J. High Energy Phys. 05 (2009) 033.

[56] S. I. Finazzo and J. Noronha, Phys. Rev. D 90, 115028 (2014).

[57] R. Yaresko and B. Kampfer, Phys. Lett. B 747, 36 (2015).

[58] D. Li, S. He, M. Huang, and Q. S. Yan, J. High Energy Phys. 09 (2011) 041.

[59] R. G. Cai, S. He, and D. Li, J. High Energy Phys. 03 (2012) 033.
[60] S. He, S. Y. Wu, Y. Yang, and P. H. Yuan, J. High Energy Phys. 04 (2013) 093.

[61] Y. Yang and P. H. Yuan, J. High Energy Phys. 11 (2014) 149.

[62] L. X. Cui, Z. Fang, and Y. L. Wu, Chin. Phys. C 40, 063101 (2016).

[63] P. Colangelo, F. Giannuzzi, S. Nicotri, and V. Tangorra, Eur. Phys. J. C 72, 2096 (2012).

[64] T. Alho, M. Jarvinen, K. Kajantie, E. Kiritsis, and K. Tuominen, J. High Energy Phys. 01 (2013) 093.

[65] T. Alho, M. Jarvinen, K. Kajantie, E. Kiritsis, C. Rosen, and K. Tuominen, J. High Energy Phys. 04 (2014) 124; 02 (2015) 033(E).

[66] M. Jarvinen, J. High Energy Phys. 07 (2015) 033.

[67] K. Chelabi, Z. Fang, M. Huang, D. Li, and Y. L. Wu, Phys. Rev. D 93, 101901 (2016).

[68] K. Chelabi, Z. Fang, M. Huang, D. Li, and Y. L. Wu, J. High Energy Phys. 04 (2016) 036.

[69] D. Li and M. Huang, J. High Energy Phys. 02 (2017) 042.

[70] S. P. Bartz and T. Jacobson, Phys. Rev. D 94, 075022 (2016).

[71] S. P. Bartz and T. Jacobson, Phys. Rev. C 97, 044908 (2018).

[72] G. S. Bali, F. Bruckmann, G. Endrodi, Z. Fodor, S. D. Katz, S. Krieg, A. Schafer, and K. K. Szabo, J. High Energy Phys. 02 (2012) 044.

[73] G. S. Bali, F. Bruckmann, G. Endrodi, Z. Fodor, S. D. Katz, and A. Schafer, Phys. Rev. D 86, 071502 (2012).

[74] J. Shen, T. Tang, and L. L. Wang, Spectral Methods: Algorithms, Analysis and Applications (Springer Science \& Business Media, Berlin, Heidelberg, 2011), Vol. 41.

[75] Y. H. Yin, A. Sun, and T. J. Wang, Spectral collocation methods for a class of nonlinear singular boundary value problems, in Proceedings of the 2013 International Conference on Advanced Mechatronic Systems, Luoyang, 2013, ( IEEE, Luoyang, China, 2013), pp. 609-612.

[76] E. V. Shuryak, World Sci. Lect. Notes Phys. 71, 1 (2004).

[77] K. A. Olive et al. (Particle Data Group Collaboration), Chin. Phys. C 38, 090001 (2014).

[78] P. Petreczky, J. Phys. G 39, 093002 (2012).

[79] R. D. Pisarski and F. Wilczek, Phys. Rev. D 29, 338 (1984).

[80] T. Bhattacharya et al., Phys. Rev. Lett. 113, 082001 (2014).

[81] E. Witten, Adv. Theor. Math. Phys. 2, 505 (1998). 